\title{
Uso de Palivizumab en Recién Nacidos Prematuros
}

Use of Palivizumab in Premature Newborns

\section{Larissa Maydelin Contreras Martínez*, Marlon}

Alexander Cerna Márquez*

\section{RESUMEN}

El Palivizumab es una terapia biológica perteneciente a la familia de los anticuerpos monoclonales IgG, indicado para la prevención de enfermedad respiratoria inferior grave causada por el Virus Sincitial Respiratorio (VSR) en neonatos prematuros con factores de riesgo. Los recién nacidos pretérminos (RNPT) se consideran los más susceptibles a dichas enfermedades en comparación con los recién nacidos a términos (RNT) sanos, lo que se traduce en mayores ingresos a Unidades De Cuidados intensivos Neonatales (UCIN), mayores costos hospitalarios y mayor morbi-mortalidad. La profilaxis con Palivizumab según la última actualización de la Academia Americana de Pediatría (AAP) se puede administrar a los recién nacidos $\geq 29$ semanas y 0 días de gestación que son menores de 12 meses en el inicio de la temporada del VSR o sean dados de alta durante ella. Para los recién nacidos durante la estación del VSR, se necesitarán al menos 5 dosis. Dada la controversia que existe actualmente en el uso de Palivizumab, por sus altos costos y discrepancias en las indicaciones relacionadas con la edad gestacional decidimos hacer una revisión bibliográfica del uso de Palivizumab en RNPT.

\section{PALABRAS CLAVE}

Prematuro, Palivizumab, Prevención, Virus Sincitial Respiratorio, Enfermedad Respiratoria.

\section{ABSTRACTS}

\footnotetext{
* Residente de 3er año de Pediatría UNAH-VS.

Dirigir correspondencia: marlon_alexander07@yahoo. com.

Recibido: 25 de Marzo 2018 Aprobado: 20 de Noviembre 2018
}

Palivizumab is a biological therapy belonging to the family of IgG monoclonal antibodies, it is indicated for the prevention of severe lower respiratory disease caused by Respiratory Syncytial Virus (RSV) in preterm infants with risk factors. Preterm infants are considered the most susceptible to severe respiratory disease by RSV compared to healthy newborns (RNT), which translates into higher incomes to Neonatal Intensive Care Units (NICU), higher hospital costs and greater morbi-mortality. Prophylaxis with Palivizumab according to the latest update of the American Academy of Pediatrics (AAP) can be administered to newborns $\geq 29$ weeks and 0 days of gestation that are less than 12 months at the start of the RSV season or are given high during it. For newborns during the RSV season, at least 5 doses will be needed. Given the controversy that currently exists in the use of Palivizumab, due to its high costs and discrepancies in the indications related to gestational age, we decided to make a bibliographic review of the use of Palivizumab in preterm infants.

\section{KEY WORDS}

Premature, Palivizumab, Prevention, Respiratory Syncytial Virus, Respiratory disease.

\section{INTRODUCCIÓN}

El Palivizumab fue aprobado en 1998 por la Food and Drug Administration (FDA) para el uso profiláctico en RNPT con o sin broncodisplasia pulmonar (DBP), tras los resultados aportados por el ensayo clínico IMpact-VSR ${ }^{(1)}$, el objetivo era disminuir la carga de enfermedad grave por VSR, reducción de las tasas y días totales de hospitalización, días de oxigenoterapia y disminución en las admisiones a UCIN por 
infecciones asociadas a este ${ }^{(2,3)}$.

Debido a las diferencias existentes entre las recomendaciones elaboradas por distintos organismos para la administración de Palivizumab como profilaxis de las infecciones por VSR en prematuros para reducir la mortalidad, la elaboración de esta revisión es para recoger información actualizada de la literatura científica y con ello aclarar las discrepancias detectadas entre las diferentes recomendaciones ${ }^{(4-10)}$.

La profilaxis con Palivizumab según la última actualización de la AAP se puede administrar a los recién nacidos $\geq 29$ semanas y 0 días de gestación que son menores de 12 meses en el inicio de la temporada del VSR o sean dados de alta durante ella. Para los recién nacidos durante la estación del VSR, se necesitarán al menos 5 dosis ${ }^{(11)}$.

Los RNPT, se consideran los más susceptibles a enfermedades respiratorias graves por el VSR en comparación con los RNT sanos ${ }^{(12)}$, lo que se traduce en mayores ingresos a UCIN, mayores costos hospitalarios y mayor morbi-mortalidad $(13,14)$.

Más de 100,000 niños son hospitalizados anualmente por VSR ${ }^{(15)}$. La bronquiolitis o neumonía asociada a VSR se considera un factor de riesgo para sibilancias recurrentes que pueden contribuir a disminuir la calidad de vida en épocas posteriores de la vida del niño ${ }^{(14)}$. La prevención de la infección se basa en medidas higiénicas y en la inmunoprofilaxis frente al VSR con Palivizumab, una inmunoglobulina monoclonal anti-VSR humanizado, aunque no existe una vacuna contra el VSR, algunos niños con alto riesgo se les da dicha profilaxis ${ }^{(14-16)}$.

Se han encontrado en algunos estudios reducción en las tasas de hospitalización, número de días de intrahospitalarios, disminución en los días de sibilancias y además una disminución en el requerimiento de oxigeno posterior a la administración de Palivizumab, aquellos que no recibieron todas las dosis recomendadas de Palivizumab tenían tasas más altas de hospitalización por enfermedad respiratoria relacionada con VSR ${ }^{(17-23)}$.
GENERALIDADES Y EPIDEMIOLOGÍA DE VSR

El VRS es la principal causa de infección del tracto respiratorio inferior grave en recién nacidos prematuros e induce daño persistentes de las vías respiratorias e hiperreactividad bronquial. La observación de que la infección por VRS infantil induce una respuesta predominantemente Th2 a alérgenos de las vías respiratorias, apoya esta sugerencia ${ }^{(24-28)}$.

Aunque el mecanismo sigue siendo desconocido, es posible que las sibilancias provocada por una infección por VRS en RNPT puede alterar la función pulmonar durante la infancia, adolescencia y la edad adulta, e incluso puede tener un efecto sobre la salud de toda la vida, con un aumento de la prevalencia de sibilancias en la primera infancia y asma. Por lo tanto, es vital para entender el impacto de la administración de Palivizumab tanto en enfermedades respiratorias durante la infancia y en la morbilidad respiratoria a largo plazo ${ }^{(16,19)}$

Algunos estudios han sugerido que la infección por VRS podría estar asociado con el asma atópica y sibilancias recurrentes ${ }^{(20)}$.

Para aquellos niños que tienen protección de anticuerpos contra el VSR adquiridos a través de la administración de Palivizumab y que también tienen la bronquiolitis, la causa puede ser un virus distinto del $\mathrm{RSV}^{(3)}$.

Las hospitalizaciones por VSR y los patrones de actividad regionales tienden a reflejar entre sí, aunque $\sim 10 \%$ de las hospitalizaciones ocurren fuera de temporada, sistemáticamente han iniciado antes y han durado más tiempo ${ }^{(15)}$.

\section{FACTORES DE RIESGO PARA INFECCIÓN POR VSR}

Aunque el VSR afecta a toda la población pediátrica, existen múltiples factores de riesgo para la infección por VSR, donde progresa más frecuentemente a las vías respiratorias inferiores donde puede provocar un cuadro de mayor gravedad, mayor necesidad oxigenoterapia, mayor número de ingresos en cuidados 
intensivos y mayor necesidad de ventilación mecánica que en la población pediátrica general. Dos estudios sirvieron para ajustar las recomendaciones enfocadas en $\mathrm{RN}$ entre la semana 32 y 35 de EG. Uno de ellos fue el estudio multicéntrico de casos y controles denominado FLIP (Factors Linked to Respiratory Syncytial Virus Infection in Premature Infants) ${ }^{(29)}$. La otra investigación fue un estudio de cohortes llamado FLIP2, el cual evaluó los factores de riesgo asociados a la infección por VSR ${ }^{(30)}$. Entre los factores de riesgo elevado son principalmente niños prematuros con EG inferior o igual a 35 semanas ${ }^{(21,31)}$, niños con neuropatías crónicas (enfermedad pulmonar crónica o displasia broncopulmonar, fibrosis quística), niños con cardiopatías congénitas, inmunodeprimidos y trasplantados ${ }^{(32,33)}$. Otros factores de riesgo son: el hacinamiento, desnutrición, edad menor de seis meses, presencia de fumadores en casa, asistencia a guarderías y ausencia de lactancia materna ${ }^{(10)}$. La población objetivo de esta revisión la constituyen los prematuros, ya que presentan mayor riesgo de contagio y de evolución grave de la infección por VSR debido a la inmadurez de las vías aéreas y la ausencia o disminución de los anticuerpos maternos. La frecuencia es 10 veces mayor que en niños nacidos a término (34). Además, la interrupción del desarrollo de los pulmones antes de las 36 SG se traduce en inmadurez del árbol bronquial y bronquiolar, menor volumen y superficie pulmonar, junto con una elasticidad reducida, flujo de aire espiratorio forzado disminuido y alteraciones en el intercambio de gases ${ }^{(35)}$. Algunos de estos pacientes prematuros han sido sometidos a algún tipo de ventilación mecánica y aspiraciones de secreciones de forma mecánica lo que a su vez puede suponer un volutrauma y barotrauma que dañan las superficies endoteliales respiratorias por microtraumatismos, lo que permite a los patógenos invadir estas superficies con mayor facilidad ${ }^{(34)}$. Se ha determinado los principales factores de riesgo de ingreso para el grupo de prematuros entre 32 y 35 semanas. Estos factores han sido analizados en estudios multicéntricos caso-control mostrando tres principales: 1) edad cronológica inferior a 10 semanas al comienzo de la estación o nacer en las 10 primeras semanas de la misma; 2) tener al menos un hermano en edad escolar o de guardería o acudir a la misma; 3) antecedente de tabaquismo materno durante la gestación. El cálculo de la utilidad predictiva de estos factores ha mostrado que la mejor predicción de ingreso por infección por VSR se presenta cuando existen los dos factores mayores (edad cronológica inferior a 10 semanas al comienzo de la estación o nacer en las primeras 10 semanas de la misma, tener al menos un hermano en edad escolar o de guardería o acudir a la misma), seguido por la combinación de un factor mayor y dos menores ${ }^{(36)}$.

\section{GENERALIDADES DEL PALIVIZUMAB}

Palivizumab una terapia biológica perteneciente a la familia de los anticuerpos monoclonales IgG, está indicado para la prevención de enfermedad respiratoria inferior grave causada por el VSR en neonatos prematuros con factores de riesgo ${ }^{(37)}$.

Representa la fusión de un anticuerpo monoclonal murino específico contra el VSR y un anticuerpo monoclonal humano no específico, compuesto por $95 \%$ de secuencias humanas y $5 \%$ de secuencias murinas sin conservación de su porción inmunogénica, por lo que no provoca la formación de anticuerpos antimurinos y debido a su parte variable posee potente actividad neutralizante frente a las cepas A y B del VSR ${ }^{(38)}$. El costo de la administración rutinaria de Palivizumab es muy elevado, lo que obliga a realizar análisis de impacto, costo-eficacia, costo-efectividad. Se administran en dosis de $15 \mathrm{mg} / \mathrm{kg}$, una vez al mes en un período de cinco meses, para prevenir la infección severa del tracto respiratorio inferior causada por el VSR ${ }^{(38-40)}$. Los efectos secundarios más comunes del Palivizumab son reacciones en el lugar de la inyección, nerviosismo y fiebre. Los efectos secundarios menos comunes incluyen diarrea y vómitos, estreñimiento, hemorragia, rinitis, problemas respiratorios, dolor, somnolencia, astenia, hiperquinesia, leucopenia y erupción (41). También se han comunicado algún caso de 
trombocitopenia, convulsiones y urticaria ${ }^{(42)}$. Se han recogido casos muy raros de anafilaxis tras la reexposición a Synagis ${ }^{\circledast}$ y casos raros de reacciones de hipersensibilidad aguda grave tras la exposición inicial o re-exposición al Palivizumab ${ }^{(43)}$. Destacar que las reacciones dérmicas y de hipersensibilidad fue uno de los motivos importantes para que no se autorizara Motavizumab ${ }^{(34)}$.

\section{USO DE PALIVIZUMAB EN PREMATUROS SANOS}

Según la última actualización 2014 de la AAP ${ }^{(22)}$, la nueva directriz no recomienda el uso rutinario de Palivizumab para lactantes sanos con una edad gestacional $(E G) \geq 29$ semanas que tengan menores de 12 meses en el inicio de la temporada del VSR o sean dados de alta durante ella, con la justificación de la recomendación de que los recién nacidos a término (RNT) o después de las 29 SG tienen una tasa de hospitalización por VSR similar a los RNT ${ }^{(44)}$. Esta nueva recomendación, más restrictiva ha generado gran controversia, ya que no pueden cambiar sustancialmente la práctica en el mundo real ${ }^{(45,46)}$. Para los recién nacidos durante la estación del VSR, se necesitarán al menos 5 dosis de Palivizumab. Los datos disponibles para los recién nacidos $\geq 29$ SG no identificaron un beneficio de la profilaxis. Los $\mathrm{RN}$ de $\leq 29$ SG pueden ser candidatos para recibir la profilaxis si tienen enfermedad cardíaca congénita (ECC), enfermedad pulmonar crónica (EPC) $\mathrm{u}$ otra condición (11). Aunque algunos expertos no recomiendan la profilaxis en este grupo etario sobre la base de que hay un pequeño aumento en el riesgo de hospitalización. La profilaxis no se recomienda en el segundo año de vida ${ }^{(11)}$. La tasa de hospitalización por VSR asociada con la administración de Palivizumab es sustancialmente menor en RNP sin DBP y sin ECC ${ }^{(47)}$.

La evidencia localizada para estudiar la efectividad, seguridad y eficiencia en los subgrupos de prematuros objetos de estudio ha sido muy escasa. Se necesitan más estudios y de mayor calidad para recomendar con alta gradación la administración de Palivizumab en prematuros menores de 29 SG, entre 6-12 meses de edad al inicio de la estación y en prematuros de 32-35 SG con factores de riesgo (2 factores de riesgo mayores; 1 factor de riesgo mayor y 2 menores $)^{(35,43,48)}$. No existe evidencia científica que apoye la administración de Palivizumab en niños prematuros menores de 29 SG entre 6-12 meses de edad al inicio de la estación para prevenir la infección por el VSR (49-52). Existe pobre evidencia científica que apoya la efectividad del Palivizumab en prematuros de 32-35 SG con factores de riesgo en cuanto a la reducción de hospitalizaciones ${ }^{(26,27,53)}$. Un único estudio de cohortes, con ciertas limitaciones metodológicas, avala su recomendación ${ }^{(54)}$. El número de factores de riesgo es un elemento fundamental a la hora de valorar la eficiencia de Palivizumab en prematuros sanos ${ }^{(55)}$. La evidencia parece indicar que al menos han de presentar dos factores de riesgo para seguir estudiando su eficiencia ${ }^{(49-50)}$. Los resultados de la eficiencia de Palivizumab en RNP de 32-35 SG no se puede afirmar que la profilaxis en este caso sea eficiente ya que no existe evidencia científica. Estudios de Palivizumab en la población en la que está indicada su administración muestran que es un medicamento seguro ${ }^{(1,53,56-58)}$. En la práctica clínica la eficacia con Palivizumab se valora por la disminución de los ingresos hospitalarios ${ }^{(59)}$. La literatura acerca de la eficiencia de Palivizumab en RNP con menos de 29 SG es muy limitada ya que no hay evidencia científica que avale la eficacia de este fármaco para esta población $(40,41,54,56,60-65)$, solo en situaciones muy restrictivas llega a ser eficiente ${ }^{(56)}$. No obstante, en relación a RNP con 32-35 SG los resultados de los estudios son muy contradictorios con unos que varían desde una rotunda falta de eficiencia ${ }^{(56)}$ y otros a una eficiencia muy elevada ${ }^{(50,51)}$, pero todos estos últimos están financiados por la industria, a excepción del informe elaborado en la AETSA ${ }^{(66)}$, cuya conclusión es la no eficiencia de Palivizumab en RNP de 32-35 SG aun considerando factores de riesgo adicionales. 
USO DE PALIVIZUMAB EN PREMATUROS CON EPC Y ECC

Palivizumab es eficaz en la prevención de la hospitalización aguda relacionada

Con VSR en bebes con factores de riesgo. La seguridad y eficacia se establecieron en niños DBP, los niños con antecedentes de parto prematuro (edad gestacional menor o igual a 35 semanas), y los niños con una cardiopatía congénita hemodinámicamente significativa “ $(47,67)$.

La profilaxis puede ser considerada durante la estación del VSR en el primer año de vida de los recién nacidos prematuros que desarrollan EPC del prematuro, definida como la edad gestacional $<32$ semanas, 0 días y necesidad de oxigeno $>21 \%$ durante al menos los primeros 28 días después del nacimiento. Durante el segundo año de vida, se recomienda la profilaxis con Palivizumab sólo para los niños que satisfacen esta definición de la EPC del prematuro y sigue requiriendo apoyo médico (corticoterapia crónica, tratamiento con diuréticos u oxígeno suplementario) durante el período de 6 meses antes del inicio de la segunda estación del VRS ${ }^{(11)}$.

Aquellos que no recibieron todas las dosis recomendadas Palivizumab tenían tasas más altas de hospitalización por enfermedad respiratoria relacionada con $\operatorname{VRS}^{(47)}$.

Los niños con cardiopatía congénita sin afectación hemodinámica con $\leq 12$ meses no puede pueden beneficiarse de la profilaxis con Palivizumab ${ }^{(44)}$.

Los niños con afectación hemodinámica significativa tienen más probabilidades de beneficiarse dela inmunoprofilaxis, en este grupo se incluyen los bebés con enfermedad cardíaca acianótica que están recibiendo medicación para controlar la insuficiencia cardíaca congestiva, que requerirán procedimientos quirúrgicos cardiacos y los lactantes con hipertensión pulmonar moderada a severa. Estas recomendaciones se aplican a los niños que califiquen en el 1er año de vida que han nacido dentro de los 12 meses siguientes al inicio de la temporada del VRS ${ }^{(54)}$.

\section{NIVELES SÉRICOS DE PALIVIZUMAB POSTERIOR A LA ADMINISTRACIÓN}

Ya que el organismo no logra crear una completa protección contra la infección por el VSR, posterior al contacto con el virus entre los cinco y quince primeros días es posible encontrar títulos adecuados de IgM persistiendo por lo menos durante tres meses. Por otro lado, anticuerpos IgG específicos antiVSR pueden detectarse en la mayoría de los casos disminuyendo significativamente un año después de la primoinfección, del mismo modo la respuesta vía IgA sérica se manifiesta después de la elevación de las IgM e IgG, y también como IgA libre en secreciones nasofaríngeas ${ }^{(68)}$.

La mayoría de los niños hospitalizados por infecciones del VSR, son menores de 6 meses de edad (69), y otro grupo que, además de la edad de riesgo, poseen patologías asociadas que la agravan como: prematuridad, compromiso cardíaco o del sistema inmune, DBP o malformaciones tóracoabdominales ${ }^{(70)}$.

En relación a los niveles de inmunoglobulina posterior a la administración de Palivizumab, se determinó que los niveles de IgG no tiene una diferencia estadísticamente significativa entre los lactantes que recibieron el tratamiento completo con Palivizumab con aquellos que recibieron el tratamiento incompleto. Sin embargo, en la IgM, el grupo que recibió las dosis completas de Palivizumab, registró un promedio mayor que los lactantes que no recibieron las dosis completas, siendo esta diferencia estadísticamente significativa $^{(38)}$.

\section{RECOMENDACIONES GENERALES PARA EL USO DEL PALIVIZUMAB}

Las recomendaciones generales para el uso de Palivizumab en niños con alto riesgo son: ${ }^{(21,71)}$

1. Niños menores de 24 meses con DBP que reciben terapia médica (oxígeno,

2. broncodilatador, diurético o terapia con corticoesteroides) para la DBP dentro de los seis meses anteriores al inicio de la estación de VSR. Estos niños deberían recibir máximo cinco dosis. 
Una vez el niño califica para iniciar la profilaxis al inicio de la estación de VSR, la administración debería continuar a través de la estación.

3. Todos los RN menores de 32 semanas ( $\leq 31$ semanas 6 días).

4. $\mathrm{RN}$ entre la semana 32 y 0 días y las 34 semanas y 6 días que tengan al menos uno de los siguientes factores de riesgo: asistir a guarderías, uno o más hermanos o vivir con otros niños menores de cinco años de forma permanente.

5. Niños con anomalía congénita de las vías aéreas o enfermedad neuromuscular.

6. Niños menores de 24 meses con cardiopatía congénita hemodinámicamente significativa, sea esta cianotisante o no.

7. Niños con inmunodeficiencia severa podrían beneficiarse de profilaxis con Palivizumab.

8. Para niños nacidos antes de la semana 32 se recomiendan hasta cinco dosis y para los mayores de esta edad gestacional se deben suministrar hasta tres dosis.

\section{DISCUSIÓN}

Los RNPT, se consideran el grupo de riesgo más vulnerable a enfermedades respiratorias graves por el VSR en comparación con los RNT sanos, lo que implica mayores ingresos a UCIN, uso de ventilación mecánica, mayores costos hospitalarios y mayor morbi-mortalidad $(13,14)$. La prevención de la infección se basa en medidas higiénicas y en la inmunoprofilaxis frente el VSR con Palivizumab (14-16). Desde la aprobación del Palivizumab en 1998 por la FDA para el uso profiláctico en RNP con o sin BDP para disminuir la infección grave por el VSR, diferentes organismos han elaborado recomendaciones para la administración de Palivizumab como profilaxis de las infecciones por VSR en prematuros para reducir la mortalidad ${ }^{(5-10,72)}$. Las indicaciones y recomendaciones de la utilización del Palivizumab en prematuros se basan en el ensayo clínico IMpact-VSR ${ }^{(1)}$, donde se obtuvo una reducción relativa del riesgo de hospitalizaciones relacionadas con el VSR del $55 \%$ y una reducción del riesgo absoluto de 5,8\%. La profilaxis con Palivizumab según la AAP se puede administrar a los $\mathrm{RN} \geq 29$ semanas que son menores de 12 meses en el inicio de la temporada del VSR o sean dados de alta durante ella. Para los recién nacidos durante la estación del VSR, se necesitarán al menos 5 dosis ${ }^{(11)}$. La Sociedad Española de Neonatología (SENeo) (73) coincide con la AAP ${ }^{(74)}$, recomendando la administración de Palivizumab en prematuros con menos de 29 SEG hasta 12 meses de edad al inicio de la estación, sobre la base que el sistema inmunitario de los prematuros menores de 29 SEG no producen IgA protectoras hasta pasados unos meses desde su nacimiento. La indicación más controvertida y donde existen mayores discrepancias en las recomendaciones de las diferentes guías clínicas se observa en los RNP de 32-35 SEG, dado el alto costo económico que supone el Palivizumab. Dos estudios sirvieron para ajustar las recomendaciones enfocadas en $\mathrm{RN}$ entre la semana 32 y 35 de EG. Uno de ellos fue el estudio multicéntrico de casos y controles denominado FLIP (Factors Linked to Respiratory Syncytial Virus Infection in Premature Infants) ${ }^{(30)}$. La otra investigación fue un estudio de cohortes llamado FLIP2, el cual evaluó los factores de riesgo asociados a la infección por VSR (31). En los últimos años se han publicado diversos estudios sobre los factores de riesgo a tener en cuenta en los RNP de 32-35 SEG para su profilaxis con Palivizumab, por lo anterior se categorizaron factores de riesgo mayores y menores, considerando la administración de Palivizumab si los niños presentaban 2 factores de riesgo mayores y un factor de riesgo mayor y 2 menores ${ }^{(75)}$. La AAP recomienda la administración de Palivizumab en niños de 32-35 SEG con factores de riesgo con edad inferior a 12 meses y que tengan hermanos menores de 5 años. Además, la AAP, recomienda la administración del anticuerpo monoclonal hasta un máximo de 3 dosis o 90 días de edad cronológica en niños de 32-35 SEG ${ }^{(74)}$. Existen discrepancias en cuanto al factor de riesgo de tener hermanos, la SENeo $2010^{(75)}$ considera un factor de riesgo mayor tener un hermano en edad escolar (hasta 14 años), basándose en los estudios FLIP y FLIP-2, la AAP considera 
factor de riesgo tener un hermano menor de 5 años, basándose en los estudios PICNIC (menor de 6 años) ${ }^{(61)}$ y de Sampalis, et al ${ }^{(76)}$. El estudio multicéntrico IMpact-VSR (1) demostró la eficacia del Palivizumab en la disminución de hospitalizaciones por VSR en una determinada población, pero existen pocos estudios de efectividad, es decir, de resultados obtenidos en la práctica clínica habitual como en el estudio de Figueras-Aloy, et al ${ }^{(55)}$. Estudios avalan la seguridad del Palivizumab para la población en la que está indicada ${ }^{(1,53,56-58)}$. La literatura acerca de la eficiencia del Palivizumab en RNP menores de 29 SEG es muy escasa, no existe evidencia científica que avalen la eficacia de este fármaco para esta población. ${ }^{(29,30,54,56,61-65)}$ Sin embargo, para la población de 32-35 SEG SG los resultados de los estudios son muy contradictorios con unos que varían desde una rotunda falta de eficiencia (77) y otros a una eficiencia muy elevada ${ }^{(50,51)}$, considerando que todos estos últimos los estudios están financiados por la industria farmacéutica.

\section{REFERENCIAS BIBLIOGRÁFICAS}

1. The IMpact-RSV Study Group. Palivizumab, a Humanized Syncytial Virus Monoclonal Antibody, Reduces Hospitalization From Respiratory Syncytial Virus Infection in High-risk Infants. [Revista en internet] 1998 [Citado, 03 marzo 2018]; 102; 531. Disponible en: http://pediatrics.aappublications.org/ content/102/3/531.full.html.

2. Resch Bernhard, Egger Beatrice, KurathKoller Stefan, Urlesberger Berndt.Respiratory hospitalizaciones por virus sincicial en los recién nacidos de 28 semanas de edad gestacional y menos en la era palivizumab. International Journal of Infectious Diseases. [Revista en internet] 2017 [Citado, 03 marzo 2018];102:531537. Disponible en: http://dx.doi.org/10.1016/j. ijid.2017.01.034

3. Byington CL and Munoz FM. Palivizumab Prophylaxis for Healthy Preterm Infants: More Data Supporting American Academy of Pediatrics
Guidelines. Pediatrics. [Revista en internet] 2016 [Citado, 07 marzo 2018];138(2);120-31. Disponible en: /content/early/2016/07/14/ peds.2016-1494.full.html.

4. Rawlings J, Rawlings V, Read J. Prevalence of low birth weight and preterm delivery in relation to the interval between pregnancies among white and black women. The New England Journal of Medicine. [Revista en internet] 1995 [Citado, 05 marzo 2018]; 332(2):69-74. Disponible en: https://www.ncbi.nlm.nih.gov/pubmed/7990903

5. Rodríguez S, Ramos R, Hernández R. Factores de riesgo para la prematurez. Estudio de casos y controles. Ginecol Obstet Mex. [Revista en internet] 2013 [Citado, 10 marzo 2018];81:499-503. Disponible en: http:// www.medigraphic.com/cgi-in/new/resumen. cgi?IDARTICULO $=45750$

6. Hübner M, Nazer J, Juárez G. Estrategias para mejorar la sobrevida del prematuro extremo. Rev Chil Pediatr. [Revista en internet] 2009 [Citado, 10 marzo 2018];80(6):551-559. Disponible en: https://scielo.conicyt.cl/scielo.php?script=sci arttext\&pid=S0370-41062009000600009

7. Palencia A. Parto Prematuro. CCAP. Ginecol Obstet Mex. [Revista en internet] 2017 [Citado, 3 marzo 2018];9(4):10-19. Disponible en: http://www.scp.com.co/precop/precop_files/ modulo_9_vin_4/Precop_9-4-B.pdf.

8. Sepúlveda A, Kobrich S, Guiñez R, Hasbun J. Morbilidad de prematuros tardíos: evidencia actual y nuevo enfoque. Rev Chil Obstet Ginecol. [Revista en internet] 2012 [Citado, 12 marzo 2018];77(2):154-158. Disponible en: https://scielo.conicyt.cl/scielo.php?script=sci_ arttext\&pid=S0717-75262012000200013

9. Raju TN, Higgins RD, Stark AR, Leveno KJ. Optimizing care and outcome for latepreterm (near-term) infants: a summary of the workshop sponsored by the National Institute of Child Health and Human Development. 
Pediatrics. [Revista en internet] 2006 [Citado, 5 marzo 2018];118:1207-14. Disponible en: http://pediatrics.aappublications.org/ content/118/3/1207?download=true

10. García-Estepa R, Navarro-Palenzuela C, Bautista-Paloma J, Romero-Tabares A, BeltránCalvo C. Efectividad, seguridad y eficiencia del palivizumab en la prevención de la infección por el virus respiratorio sincitial en prematuros. Sevilla: Agencia de Evaluación de Tecnologías Sanitarias de Andalucía. [Revista en internet] 2014 [Citado, 15 abril 2018];75(3):103-135. Disponible en: https://www.researchgate. net/publication/273074553_Efectividad_ seguridad_y_eficiencia_del_palivizumab_ en_la_prevencion_de_la_infeccion_por_el_ virus_respiratorio_sincitial_en_prematuros_ Effectiveness_safety_and_fficiency_of_ palivizumab_for_prevention_r

11. Commitee on infectious diseases and bronchiolitis guidelines commitee. Update Guidance for Palivizumab Prophylaxis Among Infants and Young Children at Increased Risk of Hospitalization for Respiratory Syncytial Virus Infection. Pediatrics. [Revista en internet] 2014 [Citado, 03 marzo 2018];134;415. Disponible en: http://pediatrics.aappublications.org/ content/134/2/415.full.html

12. Prais D, Shonfeld T, Amir J. Admision to the Intensive Care Unit for Respiratory Syncytial Virus Bronchiolitis: A National Survey Before Palivizumab Use. Pediatrics. [Revista en internet] 2003 [Citado, 03 marzo 2018];112(3);548-52. Disponible en: /content/112/3/548.full.html 13. Torchin $\mathrm{H}$, et al. Palivizumab administration in preterm infants in France: EPIPAGE-2 cohort study. Archives de Pediatrie. [Revista en internet] 2018 [Citado, 13 marzo 2018];23(5):12334. Disponible en: https://doi.org/10.1016/j. arcped.2017.12.009

14. Mercado LP et al. La eficiencia (costeefectividad) de Palivizumab como profilaxis para la infección por virus respiratorio Sincitial en prematuros de 32-35 semanas en España. An Pediatr (Barc). [Revista en internet] 2006 [Citado, 16 abril 2018];65(4):316-24. Disponible en: https://www.sciencedirect.com/r/?targetURL $=\% 2$ Fscience $\% 2$ Farticle $\% 2$ Fpii $\% 2 F S 1695403306$ $70203 \mathrm{X} \% 2 \mathrm{Fpdf}$

15. Glick FA, Kjelleren S, Hofstetter, Subramony A. RSV Hospitalizations in Comparison With Regional RSV Activity and Inpatient Palivizumab Administration, 2010-2013. Hospital Pediatrics. [Revista en internet] 2017 [Citado, 06 abril 2018];7(5);271-78. Disponible en: https://www. ncbi.nlm.nih.gov/pubmed/28381595

16. Mochizuki $\mathrm{H}$ et al. Palivizumab prophylaxis in preterm infants and subsequent wheezing: 6 Year Follow up Study. American Thoracic Society. [Revista en internet] 2017 [Citado, 16 marzo 2018];18(2);36-42. Disponible en: https:// www.ncbi.nlm.nih.gov/pubmed/28152315

17. American Academy of Pediatrics Committee on Infectious Diseases; American Academy of Pediatrics Bronchiolitis Guidelines Committee. Updated guidance for Palivizumab prophylaxis among infants and young children at increased risk of hospitalization for respiratory syncytial virus infection. Pediatrics. [Revista en internet] 2014 [Citado, 03 abril 2018];134(2):415-420. Disponible en: https://www.ncbi.nlm.nih.gov/ pubmed? $\mathrm{cmd}=$ Link\&dbFrom=PubMed \&from uid $=25070315$

18. Meissner HC, Kimberlin DW. RSV immunoprophylaxis: does the benefit ustify the cost? Pediatrics. [Revista en internet] 2013 [Citado, 06 marzo 2018];132(5):915-918. Disponible en: http://pediatrics.aappublications. org/content/early/2013/10/09/peds.2013-2449

19. Olicker A et al. Have Changing Palivizumab Administration Policies Led to More Respiratory Morbidity in Infants Born at 32-35 Weeks?. The Journal Of Pediatrics. [Revista en internet] 2015 [Citado, 16 marzo 2018];10(4);1-7. Disponible en: https://www.ncbi.nlm.nih.gov/ 
pubmed/26724119

20. Tang KP. Palivizumab prophylaxis in preterm infants. Lancet Respir Med. [Revista en internet] 2017 [Citado, 26 marzo 2018];26(17):415-20. Disponible en: http://dx.doi.org/10.1016/S22132600(17)30050-4

21. Restrepo N, Moreno YJ. Prematurez y uso de Palivizumab en prevención de infección grave por Virus Sincitial Respiratorio. Rev Medica Sanitas. [Revista en internet] 2014 [Citado, 22 abril 2018];17(3):150-158. Disponible en: http://www. unisanitas.edu.co/Revista/52/prematurez\%20 _y_uso_palivizumab_PDF.pdf

22. Burgos J. Edición Servicio de Neonatología Hospital Clínico Universidad de Chile. 1 ed. Santiago de Chile. 2001.

23. Inguanzo O, Valdés B, Iturriagagoitia E, García L,HernándezM.Caracterización deldesarrollodel pretérmino en el primer año de vida. Rev Ciencias Médicas. [Revista en internet] 2012 [Citado, 11 marzo 2018];16(1):146-157. Disponible en: $\quad$ http://scielo.sld.cu/scielo.php?script $=$ sci_ arttext\&pid=S1561-31942012000100016

24. Rellan S, García C, Aragón M. El recién nacido prematuro. Protocolos Diagnóstico Terapéuticos de la AEP. Neonatología. [Revista en internet] 2008 [Citado, 13 marzo 2018];11(5):6877. Disponible en: https://www.aeped.es/sites/ default/files/documentos/8_1.pdf

25. Nacidos Demasiado Pronto. Grupo Redactor del Resumen Ejecutivo, Kinney, M.V., Howson, C.P, McDougall, L., \& Lawn, J.E. Resumen ejecutivo de Nacidos Demasiado Pronto: Informe de Acción Global sobre Nacimientos Prematuros. March of Dimes, PMNCH, Save the Children, Organización Mundial de la Salud. [Revista en internet] 2012 [Citado, 07 abril 2018]. Disponible en: http://www.who.int/maternal_ child_adolescent/documents/born_too_soon/ es/
26. OMS (Organización Mundial de la Salud). [Internet].Tasa de nacimientos prematuros. [Actualizado 19 febrero 2018; Citado 12 abril 2018]. Disponible en: http://www. who.int/pmnch/media/news/2012/2010_ pretermbirthsper100births_es.pdf

27. Mayorga GLD et al. Perfil epidemiológico y factores de riesgo en recién nacidos prematuros, Hospital Regional, Gracias, Lempira. Rev Med Hondur. [Revista en internet] 2012 [Citado, 17 abril 2018];80(4):145-52. Disponible en: http:// www.bvs.hn/RMH/pdf/2012/pdf/Vol80-4-20123.pdf

28. Hoffman M, Kling Michele, Kieman Mike. El nacimiento Prematuro es ahora la Principal causa de muerte en niños pequeños. UNICEF. [Revista en internet] 2013 [Citado, 17 abril 2018];25(3):124-29. Disponible en: https://www. unicef.org/venezuela/spanish/Dia_Mundial_ del_Nacimiento_Prematuro_CdP_Conjunto.pdf

29. Figueras J, Carbonell X, Quero J. Case Control study of the risk factor linked to respiratory syncytial virus infection requiring hospitalization in premature infants born at a gestational age of 33-35 weeks in Spain. Pediatr Infect Dis J. [Revista en internet] 2004 [Citado, 17 abril 2018]; 23(3):815-20. Disponible en: https:// www.ncbi.nlm.nih.gov/pubmed/15361718

30. Figueras J, Carboney X, Quero J, Fernández B, Guzmán J, Echaniz I, et al. FLIP-2 Study: Risk Factors Linked to Respiratory Syncytial Virus Infection Requiring Hospitalization in Premature Infants Born in Spain at a Gestational Age of 32 to 35 Weeks. Pediatr Infect DisJ. [Revista en internet] 2008 [Citado, 20 abril 2018];27(6):78893. Disponible en: https://www.ncbi.nlm.nih. gov/pubmed/18664927 